Article

\title{
Distance-Constrained Outage Probability Analysis for Device-to-Device Communications Underlaying Cellular Networks with Frequency Reuse Factor of 2
}

\author{
Devarani Devi Ningombam and Seokjoo Shin * \\ Department of Computer Engineering, Chosun University, 309 Pilmun-daero, Gwangju 61452, Korea; \\ devaraninin@gmail.com \\ * Correspondence: sjshin@chosun.ac.kr; Tel.: +82-62-230-6030
}

Received: 10 September 2018; Accepted: 5 October 2018; Published: 6 October 2018

\begin{abstract}
Device-to-device (D2D) communication is affirmed as one of the dynamic techniques in improving the network throughput and capacity and reducing traffic load to the evolved Node $B$ (eNB). In this paper, we propose a resource allocation and power control technique in which two-pairs of D2D users can simultaneously share same uplink cellular resource. In this case, interference between D2D users and cellular users is no longer insignificant so it must be properly handled. The proposed scheme considers fractional frequency reuse (FFR) scheme as a promising method that can relatively reduce the intra-cell interference. The main objective of the proposed scheme is to maximize the D2D communication throughput and overall system throughput by minimizing outage probability. Hence, we formulate an outage probability problem and overall system throughput optimization problem while guaranteeing minimum allowable signal-to-interference-plus-noise ratio (SINR). For fair distribution of cellular resources to multiple D2D pairs, we used Jain's fairness index (JFI) method. Simulation is conducted in MATLAB and our simulation results demonstrate that the proposed scheme achieves remarkable system performance as compared with existing methods.
\end{abstract}

Keywords: device-to-device communication; fractional frequency reuse; outage probability; Jain's fairness index; throughput

\section{Introduction}

With the expansion of mobile multimedia services, the amount of traffic in the network has been explosively increasing. To accommodate the avalanche of traffic, there is a need to maximize the capacity of the cellular network. However, the available radio spectrum for cellular networks is very scarce and needs to be utilized in the most efficient way [1]. In recent years, the popularity of device-to-device (D2D) communications in cellular networks has been increasing more rapidly [2]. D2D communication addresses to the direct communication between multiple devices without passing data traffic to an evolved Node B (eNB). It can operate in licensed or unlicensed spectrum and supports reusing of the frequency band of the operational cellular network. An extensive survey on D2D communication in cellular networks has been presented in [3]. In the paper, the authors explained two classes for D2D communications, namely inband and outband. Moreover, the study also categorized different types of D2D communications based on the spectrum-sharing phenomenon between cellular users and D2D users: underlay and overlay. In the underlay D2D, cellular users and D2D users operate at the same frequency band. Therefore, it can accommodate more users on a given frequency band. While the overlay D2D allows cellular users and D2D users operate at two different frequency bands, this method reduces the amount of achievable data rate for cellular communications. The most popular benefits offered by D2D communication include improved 
spectral efficiency and throughput and reduced latency. In spite of various advantages provided by the D2D communication, it introduces various challenges on the communication system. Two important challenges are: inter-cell interference generated by D2D transmitter to the cellular user and interference from the cellular user to the D2D receiver [4]. The presence of interference could result in performance degradation of cellular and D2D communications system. Therefore, there is a need for an effective solution that guarantees D2D communications will not generate deleterious interference to conventional cellular users. A thorough review on different interference mitigation schemes has been employed in [5]. The main challenges introduced due to the integration of D2D in conventional cellular networks, namely device discovery, mode selection, resource management, security, etc., have been discussed thoroughly. From their investigation, the authors concluded that the multi-antenna beamforming with power control technique minimizes intra-cell and inter-cell interference in the network. In [6], the authors proposed an intelligent resource management scheme for D2D communications in heterogeneous networks in which the network-assisted devices are controlled based on the specific information broadcast. However, in this scheme, the power control method was not considered. A power control method for D2D communication underlaying uplink cellular networks was studied in [7]. In this study, the performance of the system was analyzed by considering both D2D and cellular communication simultaneously. Comparing the result of the proposed scheme with conventional cellular communication demonstrates the enhancement of system throughput. In [8], a problem of joint resource block and power allocation in D2D communications underlaying uplink cellular networks was discussed. The study analyzed the optimization problem to reduce the total interference of the cellular and D2D links in the system. The results showed that their proposed scheme improves the sum data rate and reduces the power consumption. The disadvantage of this study is: an omni-directional antenna was assumed for cellular users and thus the proposed scheme is not spectrally efficient.

Therefore, we can achieve higher spectrum efficiency by considering fractional frequency reuse (FFR) technique, where the whole cell area is divided into two non-overlapping regions, namely inner cell and edge cell regions [9]. Furthermore, each region of the cell is sectorized into three equivalent sections using directional antennas. A joint resource allocation and power control scheme using FFR technique for underlay D2D communications was presented in [10]. The authors discussed on distance-based resource allocation scheme for a single cell system model and formulate throughput optimization problem. The results showed that their proposed scheme improves the throughput in comparison with the random resource allocation scheme. However, the paper considered the resource reuse scenario for frequency reuse factor (FRF) of 1, which is not spectrally efficient. In [11], outage probability analysis for D2D communications in FRF of 1 network was discussed. In the paper, the author proposed a location-based outage probability analysis of D2D communications for a multi-cell network. However, for a fast growing demand for high data rate services, there is need to further increase FRF. Fairness allocation of cellular resources among the D2D pairs is an important aspect for underlay D2D communications wherein D2D pairs coexist with cellular users. The authors in [12] proposed a novel resource allocation scheme for D2D communications using FFR and soft frequency reuse (SFR) methods to enhance the coverage probability of the network. Also, the power control method was introduced to analyze the trade-off between the power consumption and performance of the system. In this study, the authors assumed the resource reuse factor of inner and outer cell regions for SFR method to be equal to two and one, respectively. Comparing the result of their scheme with that of unity frequency reuse scheme proves the improvement in system capacity. The disadvantage of this scheme is that the inter-cell interference coordination function is not considered. A proportional fairness-based resource allocation and power control scheme was presented in [13]. In the paper, the authors analyze the time-varying feature of the user's channel condition to maximize the throughput and fairness of the system.

The remainder of this paper is organized as follows. In Section 2, related works are discussed. Our contribution in this paper is provided in Section 3. Section 4 explains the system model. In Section 5, the 
proposed scheme is presented. Section 6 provides performance analysis. Finally, Section 7 concludes the paper.

\section{Related Work}

Nowadays, the concern of the interference caused by the D2D communication underlaying uplink cellular network has been the target of many researchers. Some of the related works are mentioned in this section.

The problem of interference mitigation in a D2D communication underlaying uplink cellular networks has been discussed in [14]. In this paper, an optimization problem is analyzed to minimize the total interference of the cellular network. The optimization problem is formulated in two steps: at the first step the resources are allocated with a fixed power level, and at the second step interference minimization is addressed into multiple interference minimization problems per available resources. The results showed that their proposed scheme reduces the total interference significantly as compared to random resource allocation (RRA) scheme. However, the proposed system model is equipped with a single omni-directional antenna. Therefore, this scheme is not spectral efficient. The authors of [15] proposed a novel scheme to find the optimal solution for D2D users by defining two types of constraints, namely the peak interference and average interference caused by the D2D communication. However, since both the cellular users and D2D pairs employed the resources non-orthogonally, intra-cell interference becomes severe. In order to mitigate intra-cell interference, a FFR scheme for D2D communications has been discussed in [16]. In the paper, the authors proposed a resource allocation (RA) scheme for D2D communications for a multi-cell cellular network with FRF of 1 . In this work, the optimization problem was formulated to reduce the outage probability and increase the overall network throughput. Also, the advantages of limited co-channel power control, fractional frequency power control, and fractional frequency adaptive power control schemes were analyzed. However, the available frequency band was not fully allocated to the whole cell area. In [17], an optimization problem was formulated to maximize the overall system throughput. The demerit of this proposed scheme is that it only focused on the performance improvement of conventional cellular users.

The work in [18] presented a thorough survey on the various fairness models in wireless networks. In this context, the authors discussed different fairness models and compared in terms of the energy consumption, power consumption, scheduling phenomenon, utility management, and resource allocation scenario. An optimal trade-off between system efficiency and Jain's fairness index (JFI) for multiple D2D users has been studied in [19]. An admissible utilization method is presented by considering the trade-off policy between system efficiency and fairness index. However, complexity of the proposed method is very high. In [20], a joint power control and fairness scheduling for D2D communication have been proposed. The Hungarian algorithm is considered to maximize the overall throughput while ensuring signal-to-interference-noise ratio (SINR) requirement. However, this method does not provide significant performance improvement as it only assumed the present time slot.

The work in [21] proposed a scheme which analyzes outage probability by jointly considering the effect of interference, noise, and Rayleigh multipath fading phenomenon. Also, the outage probability model is analyzed using additive white Gaussian noise based on stochastic geometry, Laplace transforms, and probability density function. Moreover, an analytical expression under realistic interference scenarios was derived. In [22], the authors introduced exact and approximate formulas for calculating outage probability. The results showed that their proposed scheme significantly improves the system throughput. The limitation of this scheme is that the system model does not accommodate large capacity of users. Therefore, it would be better to consider resource allocation and power control scheme with FFR technique for FRF of 2 which can achieve spectral efficiency and increase the capacity of users to the network. 


\section{Contributions}

Comprehensive research on D2D communications has been administered using FFR scheme for sharing resources between the cellular users and D2D users. Motivated by the literature works, we propose a resource allocation and power control technique for D2D communications with FRF of 2, where the locations of the D2D users are assume to be static. Hence, the contributions of this paper are summarized as below.

- We introduce a novel resource allocation algorithm, which supports frequency reuse factor of 2 .

- We analyze the outage probability of D2D pairs and overall system with respect to distance between D2D-Rx and eNB, SINR threshold, and number of available D2D pairs. The proposed scheme provides lowest outage probability as compared with other existing schemes.

- We improve the D2D throughput and overall system throughput with less computational complexity.

- We minimize the average energy consumption of the system with varying number of available D2D pairs.

- We discuss the fairness distribution of resources by considering Jain's fairness index.

To the best of our knowledge, this is the first work that proposes resource allocation and power control scheme based on the fairness index by cell sectorization mechanism for FRF of 2 . The formulation in this paper yields tractable results that can be applicable in future cellular networks.

\section{System Model}

Consider a D2D communication underlaying LTE-A uplink cellular network, where $D=\{1,2, \ldots ., M\}$ D2D users and $C=\{1,2, \ldots \ldots, N\}$ cellular users are uniformly distributed in a cell as shown in Figure 1 . A D2D transmitter and its predefined receiver form a D2D pair. For simplicity we assumed a single cell scenario with eNB located at the center of the cell. The probability density function (PDF) of a D2D pair is defined as:

$$
f_{i}(l)=\frac{2 l}{\left(R_{i}\right)^{2}}, 0<l \leq R_{i}
$$

The complementary cumulative distribution function $(\mathrm{CDF})$ is expressed by:

$$
F_{i}(l)=\int_{l}^{R_{i}} f_{i}(l) d l=1-\left(\frac{l}{R_{i}}\right)^{2}
$$

where $R_{i}$ is the $\mathrm{D} 2 \mathrm{D}$ communication range.

As shown in the Figure 1, eNB is equipped with three $120^{\circ}$-directional antennas and assumed that the density of cellular users is greater than that of the D2D users in a cell. There are two main types of uplink interference generated due to D2D communications. In Figure 1a, signal transmitted from cellular user causes interference to D2D-Rx, and Figure $1 \mathrm{~b}$ shows the interference generated by D2D-Tx to eNB. To mitigate the interference, we consider cell sectorization scheme in which cell area is divided into two non-overlapping regions, namely: inner and edge region. Cellular users and D2D pairs existing in inner cell region are named as $N_{i n}$ and $M_{i n}$, respectively. Similarly, cellular users and D2D pairs existing in edge cell region are named as $N_{\text {edge }}$ and $M_{\text {edge }}$, respectively. The locations of the both users are modeled based on the distance of the users from the eNB. Furthermore, each region is subdivided into three equivalent sub-sections. Therefore, the entire frequency spectrum is divided into six sub-bands and different sub-bands are allotted to each section of the cell. It is important to note that there is no interference among cellular users since the available resources are orthogonally assigned to all the cellular users by the eNB.

For general realization, the received signal power from the eNB at the cellular user and D2D receiver is expressed as:

$$
P_{\text {rec }}=P_{T} d^{-\alpha}|h|^{2}
$$


where $P_{T}$ is the transmit power, $d$ is the distance between transmitter and receiver, and $h$ is the channel coefficient.

Therefore, the received signal of a typical user is given by:

$$
r(t)=r_{A 1}(t)+\sum_{n e \neq A 1} r_{n e}(t)+x(t)
$$

where $r_{A 1}(t)$ is the received signal from the desired user in a section of the cell, $r_{n e}(t)$ is the sum of signals received from neighboring sections of the cell, and $x(t)$ is the noise with power spectral density $P_{N}$.

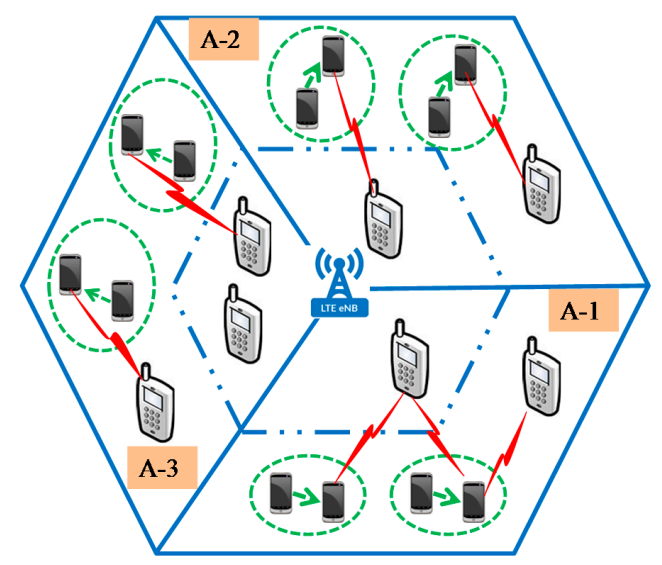

(a)

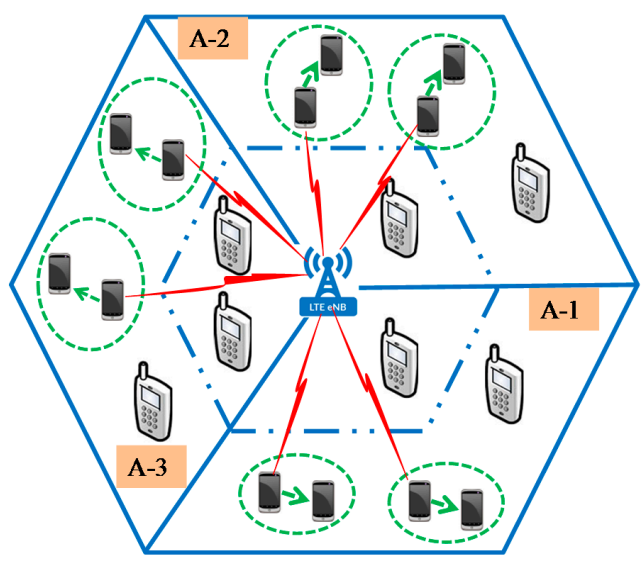

(b)

Figure 1. Uplink interference scenarios: (a) interference from cellular user to D2D-Rx and (b) interference from D2D-Tx to eNB. D2D, device-to-device; eNB, evolved Node B.

\section{Proposed Scheme}

In the proposed scheme, we assume that the D2D pairs in inner cell region reuse the resources of cellular users in the edge cell region and vice versa. However, the reuse of cellular resources by the D2D pairs in the same section of the cell is not allowed. The main objective of the proposed scheme is to mitigate the interference generated due to deployment of D2D communication into conventional cellular networks. In this section, we provide an analytical expression for interference caused by D2D communications while reusing the same cellular resource by two-pairs of D2D simultaneously. The operational flow of the proposed resource allocation scheme with FFR of 2 is presented in Algorithm 1.

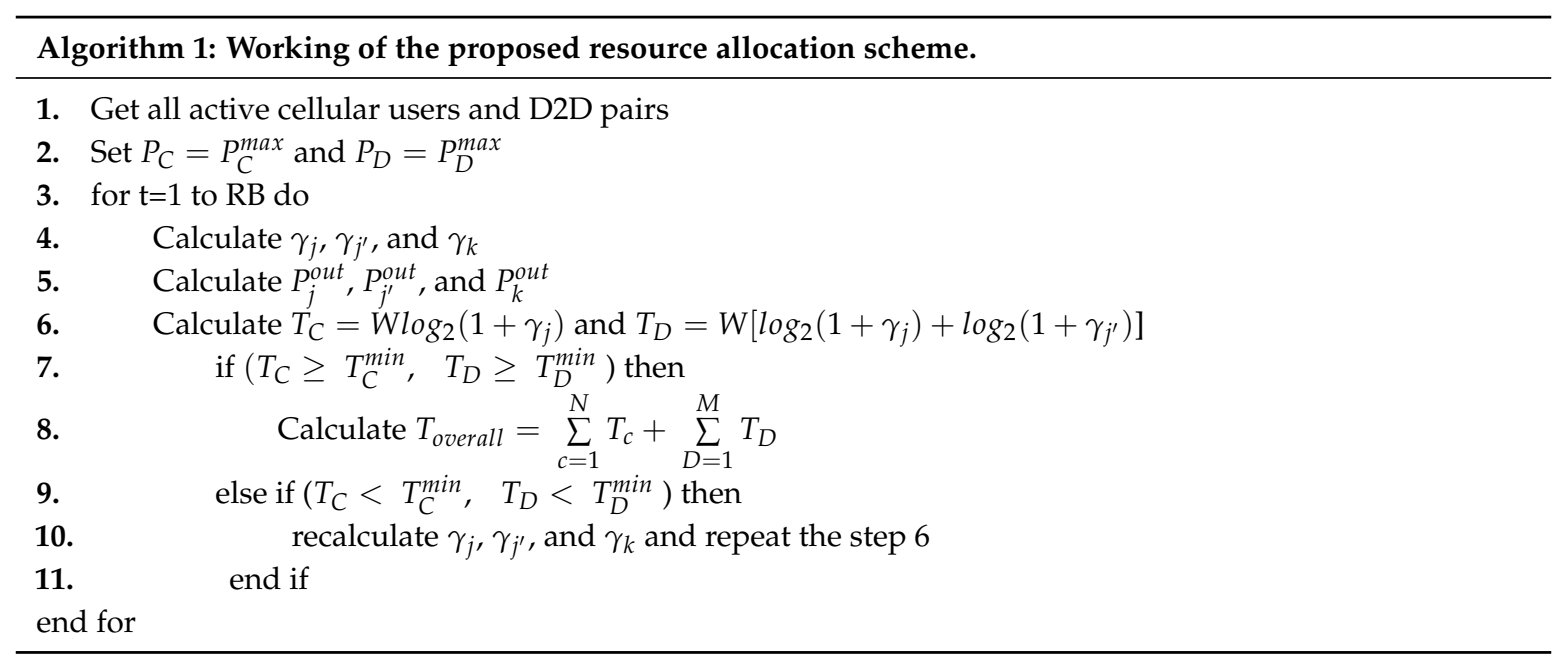




\subsection{Formulation of Signal-to-Interference-Plus-Noise Ratio (SINR)}

SINR is a commonly used for the analysis of characteristic of a communication system. The general expression for SINR constraint for a device can be written as

$$
\gamma \geq \gamma^{T h}
$$

where $\gamma^{T h}$ is the predefined SINR threshold.

A signal transmission is said to be successful when the received SINR of the user is greater than or equal to the predefined threshold SINR. To derive the SINR distribution, we assume that the resource of a cellular user $k$ exists in inner cell region of A-1 (shown in Figure 1) is reused by two-pairs of D2D existing in edge cell region of A-1. We denote $P_{i}$ as the transmit power of D2D-Tx $i, P_{i^{\prime}}$ as the transmit power of D2D-Tx $i^{\prime}, P_{k}$ as the transmit power of cellular user $\mathrm{k}, l_{i, B}$ as the distance between D2D-Tx $i$ and eNB, $l_{i^{\prime}, B}$ as the distance between D2D-Tx $i^{\prime}$ and eNB, $l_{k, j}$ as the distance between cellular user $k$ and D2D-Tx $j, l_{k, j^{\prime}}$ as the distance between cellular user $k$ and D2D-Tx $j^{\prime}$, and $l_{k, B}$ as the distance between cellular user $k$ and eNB. Let $h_{i, B}$ and $h_{i^{\prime}, B}$ represent the channel coefficients from eNB to D2D-Tx $i$ and D2D-Tx $i^{\prime}$, respectively, $h_{k, j}$ and $h_{k, j^{\prime}}$ represent the channel coefficients from cellular user $k$ to D2D-Tx $i$ and D2D-Tx $i^{\prime}$, respectively, and $h_{k, B}$ represent the channel coefficients from eNB to cellular user $k$. Moreover, we define the interference from D2D-Tx $i$ to D2D-Rx $j^{\prime}$ and from D2D-Tx $i^{\prime}$ to D2D-Rx $j$ by $I_{i^{\prime}}$ and $I_{i}$, respectively, the interference from neighboring D2D-Tx to D2D-Rx $j$ and $j^{\prime}$ by $I_{j, n e}$ and $I_{j^{\prime}, n e}$, respectively.

Therefore, the received SINRs of D2D-Rx $j$ and $j^{\prime}$ are given by:

$$
\begin{gathered}
\gamma_{j}=\frac{P_{i} \cdot l_{i, B}^{-\alpha} \cdot\left|h_{i, B}\right|^{2}}{P_{k} \cdot l_{k, j}^{-\alpha} \cdot\left|h_{k, j}\right|^{2}+I_{i^{\prime}}+\sum_{n e \neq A 1} I_{j, n e}+P_{N}} \\
\gamma_{j^{\prime}}=\frac{P_{i^{\prime}} \cdot l_{i^{\prime}, B}^{-\alpha} \cdot\left|h_{i^{\prime}, B}\right|^{2}}{P_{k} \cdot l_{k, j^{\prime}}^{-\alpha} \cdot\left|h_{k, j^{\prime}}\right|^{2}+I_{i}+\sum_{n e \neq A 1} I_{j^{\prime}, n e}+P_{N}}
\end{gathered}
$$

respectively, where $\alpha$ is the path loss coefficient and $P_{N}$ is the noise power.

Similarly, the received SINR of cellular user $k$ is given by:

$$
\gamma_{k}=\frac{P_{k} \cdot l_{k, B}^{-\alpha} \cdot\left|h_{k, B}\right|^{2}}{P_{i} \cdot l_{i, B}^{-\alpha} \cdot\left|h_{i, B}\right|^{2}+P_{i^{\prime}} \cdot l_{i^{\prime}, B}^{-\alpha} \cdot\left|h_{i^{\prime}, B}\right|^{2}+\sum_{n e \neq A 1} I_{B, n e}+P_{N}}
$$

where $I_{k, n e}$ is the interference from the neighboring D2D-Tx to the eNB.

Here, the analytical expressions for different interference are expressed as:

$$
\begin{gathered}
I_{i^{\prime}}=P_{i^{\prime} \cdot} \cdot l_{i^{\prime}, j}^{-\alpha} \cdot\left|h_{i^{\prime}, j}\right|^{2} \\
I_{i}=P_{i} \cdot l_{i, j^{\prime}}^{-\alpha} \cdot\left|h_{i, j^{\prime}}\right|^{2}, \\
I_{j, n e}=P_{n e} \cdot l_{n e, j}^{-\alpha} \cdot\left|h_{n e, j}\right|^{2} \\
I_{j^{\prime}, n e}=P_{n e} \cdot l_{n e, j^{\prime}}^{-\alpha} \cdot\left|h_{n e, j^{\prime}}\right|^{2} \\
I_{B, n e}=P_{n e} \cdot l_{n e, B}^{-\alpha} \cdot\left|h_{n e, B}\right|^{2} .
\end{gathered}
$$


In order to determine the proper set of cellular resources that can be reuse by D2D pairs, we formulate a channel coefficient relationship as listed below [23].

$$
\left.\begin{array}{c}
\delta_{i}=\frac{\left|h_{k, B}\right|^{2}}{\left|h_{k, j}\right|^{2}} \cdot \frac{\left|h_{i, j}\right|^{2}}{\left|h_{i, B}\right|^{2}} \\
\delta_{i^{\prime}}=\frac{\left|h_{k, B}\right|^{2}}{\left|h_{k, j^{\prime}}\right|^{2}} \cdot \frac{\left|h_{i^{\prime}, j^{\prime}}\right|^{2}}{\left|h_{i^{\prime}, B}\right|^{2}}
\end{array}\right\}
$$

where $\delta_{i}$ and $\delta_{i^{\prime}}$ are the factors that determine the channel gain for two D2D pairs. When the value of $\delta_{i}$ and $\delta_{i^{\prime}}$ are high, the system can achieve highest throughput after integrating D2D communication into conventional cellular network.

\subsection{Formulation of Outage Probability (Pout)}

Outage probability is defined as the probability that the SINR drops below a predefined threshold SINR value [24]. The general expression for outage probability is defined as:

$$
P^{\text {out }}=\operatorname{Pr}_{r}\left[\gamma \leq \gamma^{T h}\right]
$$

where $\gamma^{T h}$ is the predefined threshold SINR value.

In the proposed scheme, we assume that the average SINR is calculated by dividing transmit power to noise power as below.

$$
\widetilde{\gamma}=\frac{\text { Transmit power of a user }}{\text { Noise power }}=\frac{P}{P_{N}}
$$

Considering Equations (5-15) and substituting $\gamma=\gamma^{T h}$, the outage probability for both D2D pairs and cellular user can be obtained as:

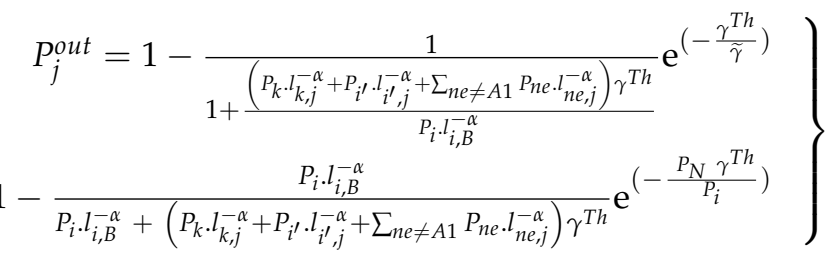

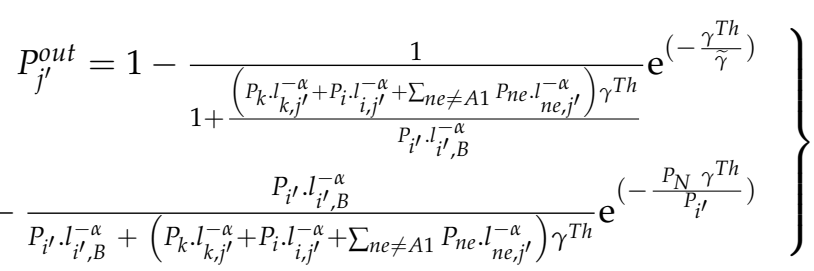

$$
\begin{aligned}
& \left.\begin{array}{c}
P_{k}^{\text {out }}=1-\frac{1}{1+\frac{\left(P_{i} \cdot l_{i, B}^{-\alpha}+P_{i^{\prime}} \cdot l_{i^{\prime}, B}^{-\alpha}+\sum_{n e \neq A 1} P_{n e} \cdot l_{n e, B}^{-\alpha}\right) \gamma^{T h}}{P_{k} \cdot l_{k, B}^{-\alpha}}} e^{\left(-\frac{\gamma^{T h}}{\tilde{\gamma}}\right)} \\
-\frac{P_{k} \cdot l_{k, B}^{-\alpha}}{P_{k} \cdot l_{k, B}^{-\alpha}+\left(P_{i} \cdot l_{i, B}^{-\alpha}+P_{i^{\prime}} \cdot l_{i^{\prime}, B}^{-\alpha}+\sum_{n e \neq A 1} P_{n e} \cdot l_{n e, B}^{-\alpha}\right) \gamma^{T h}} e^{\left(-\frac{P_{N} \gamma^{T h}}{P_{k}}\right)}
\end{array}\right\}
\end{aligned}
$$

\subsection{Channel Model}

In order to determine the user's connection behavior, a path-loss model for an urban environment is considered in this paper. We apply different path-loss models with zero mean and unit variance log-normal fading to D2D users and cellular users. The distance-dependent path-loss models for line of sight (LOS) communications for D2D users and cellular users are expressed as [25]: 


$$
\left.\begin{array}{c}
P L_{i}=49+40 \log _{10}\left(\frac{l_{i, j}}{1000}\right)+30 \log _{10}\left(\frac{f_{c}}{1000}\right) \\
P L_{i^{\prime}}=49+40 \log _{10}\left(\frac{l_{i^{\prime}, j^{\prime}}}{1000}\right)+30 \log _{10}\left(\frac{f_{c}}{1000}\right)
\end{array}\right\}
$$

where $l_{i, j}, l_{i^{\prime}, j^{\prime}}$, and $l_{k}$ are the distance from D2D-Tx $i$ to D2D-Rx $j$, from D2D-Tx $i^{\prime}$ to D2D-Rx $j^{\prime}$, and from cellular transmitter to receiver, respectively, and $f_{c}$ is the carrier frequency in $\mathrm{GHz}$.

\subsection{Resource Sharing Optimization}

The main objective of the proposed scheme is to maximize the overall system throughput compared to other existing schemes. In order to evaluate the throughput of cellular users and D2D pairs, we consider mathematical expression of Shannon's formula. Therefore, throughput of the overall system is defined as:

$$
T_{\text {overall }}=\sum_{c=1}^{N} T_{c}+\sum_{D=1}^{M} T_{D}
$$

where $T_{\mathcal{C}}$ and $T_{D}$ are the throughput of cellular user and D2D pairs, respectively, which is defined by:

$$
\begin{gathered}
T_{c}=W \log _{2}\left(1+\gamma_{k}\right) \\
T_{D}=W\left[\log _{2}\left(1+\gamma_{j}\right)+\log _{2}\left(1+\gamma_{j^{\prime}}\right)\right]
\end{gathered}
$$

where $W$ is the channel bandwidth.

Therefore, the throughput maximization problem can be formulated by:

$$
\left.\max _{c \in N, D \in M}\left(\sum_{c=1}^{N} T_{c}+\sum_{D=1}^{M} T_{D}\right)\right\}
$$

subject to

$$
\left.\begin{array}{rl}
P_{C}^{\min } \leq & P_{C} \leq P_{C}^{\text {max }} ; P_{D}^{\min } \leq P_{D} \leq P_{D}^{\max }, \forall C \in N, D \in M \\
\gamma_{C}^{\text {min }} \leq \gamma_{C} ; \gamma_{C}^{\min } \leq \gamma_{C}, \forall C \in N, D \in M
\end{array}\right\}
$$

where $\gamma_{C}^{m i n}$ and $\gamma_{D}^{m i n}$ are the minimum allowable SINRs of the cellular user and D2D pair respectively. $P_{C}^{\min }$ and $P_{C}^{\max }$ are the minimum and maximum power range for the cellular user, and $P_{D}^{\min }$ and $P_{D}^{\max }$ are the minimum and maximum power ranges of the D2D pair. Equation (26) ensures that the transmit power of both cellular users and D2D pairs should be within the minimum and maximum transmit power range. This is the arbitration between system capacity and interference. Simillarly, Equation (26) ensures that the SINR of both cellular users and D2D pairs should be greater than or equal to minimum SINR threshold. Hence, the upper and lower bounds transmit power of cellular user should satisy the following criteria.

$$
P_{C}^{\min } \leq \frac{\gamma_{k}\left(P_{i} \cdot l_{i, B}^{-\alpha} \cdot\left|h_{i, B}\right|^{2}+P_{i^{\prime}} \cdot l_{i^{\prime}, B}^{-\alpha} \cdot\left|h_{i^{\prime}, B}\right|^{2}+\sum_{n e \neq A 1} I_{B, n e}+P_{N}\right)}{l_{k, B}^{-\alpha} \cdot\left|h_{k, B}\right|^{2}} \leq P_{C}^{\max }
$$

Similarly, the upper bound and lower bounds transmit power of both D2D-Rxs are derived as:

$$
P_{D}^{\min } \leq P_{i}, P_{i^{\prime}} \leq P_{D}^{\max }
$$




$$
\begin{gathered}
P_{D}^{\min } \leq \frac{\gamma_{j}\left(P_{k} \cdot l_{k, j}^{-\alpha} \cdot\left|h_{k, j}\right|^{2}+I_{i^{\prime}}+\sum_{n e \neq A 1} I_{j, n e}+P_{N}\right)}{P_{i} \cdot l_{i, B}^{-\alpha} \cdot\left|h_{i, B}\right|^{2}} \\
\frac{\gamma_{j^{\prime}}\left(P_{k} \cdot l_{k, j^{\prime}}^{-\alpha} \cdot\left|h_{k, j^{\prime}}\right|^{2}+I_{i}+\sum_{n e \neq A 1} I_{j^{\prime}, n e}+P_{N}\right)}{P_{i^{\prime} \cdot} \cdot l_{i^{\prime}, B}^{-\alpha} \cdot\left|h_{i^{\prime}, B}\right|^{2}} \leq P_{D}^{\max }
\end{gathered}
$$

\subsection{Jain's Fairness Index (JFI)}

In this section, fairness allocation is depicted as a special case of resource allocation scheme. As the proposed scheme considers distributed resource allocation mechanism, the fairness index is analyzed based on the local information of the D2D pairs. To evaluate the fairness allocation of cellular resource among D2D pairs, JFI is use in the proposed scheme. JFI is expressed as below [13].

$$
J F I\left(T_{D}\right)=\frac{\left(\sum_{D=1}^{M} T_{D}\right)^{2}}{(M) \cdot \sum_{D=1}^{M} T_{D}^{2}}, \forall D \in M
$$

From Equation (24), we have,

$$
\left.\begin{array}{rl}
J F I\left(T_{D}\right)= & \frac{\left(\sum_{D=1}^{M} W\left[\log _{2}\left(1+\gamma_{j}\right)+\log _{2}\left(1+\gamma_{j^{\prime}}\right)\right]\right)^{2}}{(M) \cdot \sum_{D=1}^{M}\left(W\left[\log _{2}\left(1+\gamma_{j}\right)+\log _{2}\left(1+\gamma_{j^{\prime}}\right)\right]\right)^{2}} \\
& =\frac{\left(\sum_{D=1}^{M}\left[\log _{2}\left(1+\gamma_{j}\right)+\log _{2}\left(1+\gamma_{j^{\prime}}\right)\right]\right)^{2}}{(M) \cdot \sum_{D=1}^{M}\left[\log _{2}\left(1+\gamma_{j}\right)+\log _{2}\left(1+\gamma_{j^{\prime}}\right)\right]^{2}}
\end{array}\right\}, \forall D \in M
$$

This Equation determines the rate of fairness of a set of users where there are total $M$ D2D users are residing. From Equation (31), it is clearly seen that $J F I\left(T_{D}\right)$ value is having an interval $\frac{1}{M}$, 1 , i.e.,

$$
J F I=\left\{\begin{array}{l}
1, \quad \text { Proposed scheme attains optimal fair allocation of resources } \\
\frac{1}{M}, \text { Proposed scheme attains least fair allocation of resources }
\end{array}\right.
$$

\subsection{Complexity Anaylis}

In order to solve the optimization problem with lower computational complexity, we consider that single cellular resource can simultaneously reuse by two D2D pairs. The complexity of the resource allocation algorithm for two D2D pairs sharing same cellular resource is denoted as $O\left(M^{2}\right) . O(N)$. A two-step approach is presented to solve this fundamental complexity. At the first step, an exhaustive search for resource reuse partner is avoided by choosing proper channel coefficient relationship, $\delta$. At the second step, we consider the conditions of upper and lower bounds transmit power. Thus, the proposed scheme is a low complexity solution suitable for realistic D2D communications environment.

\section{Performance Analysis}

\subsection{Simulation Parameters and Values}

To evaluate the performance of the proposed resource allocation scheme with FFR technique, we apply both mathematical analysis and conduct extensive simulations on Monte Carlo simulation. A single cell scenario is assumed with radius of $600 \mathrm{~m}$ and the eNB located at the center of the cell. In each realization, cellular users and D2D-Txs are generated randomly with uniform distribution over the entire cell area. The distances between D2D-Tx and its corresponding D2D-Rx are considered uniformly distributed in the range between 1 and $50 \mathrm{~m}$. In this paper, the transmit powers of cellular user and D2D user are set at $20 \mathrm{dBm}$ and $15 \mathrm{dBm}$, respectively. The corresponding number of resource blocks is chosen in accordance with the bandwidth defined by the LTE standard for a single component carrier. The main simulation parameters are presented in Table 1. Other simulation parameters are 
chosen with respect to 3GPP LTE regulation [26] and simulation results are averaged through the specified iterations.

Table 1. Simulation parameters and values.

\begin{tabular}{ll}
\hline Simulation Parameters & Values \\
\hline Cell outline & Hexagonal cell framework \\
Total number of resource blocks & $50[26]$ \\
Cellular user transmission power & $20 \mathrm{dBm}$ \\
D2D transmission power & $15 \mathrm{dBm}$ \\
Antenna type & $3-120^{\circ}$ directional antennas \\
Noise Power & $-174 \mathrm{dBm} / \mathrm{Hz}$ \\
D2D pair distance & $1-50 \mathrm{~m}$ \\
Minimum device distance from eNB & $10 \mathrm{~m}$ \\
Carrier frequency & $2 \mathrm{GHz}$ \\
Uplink bandwidth & $10 \mathrm{MHz}[26]$ \\
Path loss exponent & 3 \\
Cell radius & $600 \mathrm{~m}$ \\
Monte Carlo simulation runs & 15,000 \\
\hline
\end{tabular}

\subsection{Simulation Results and Discussion}

We compare our proposed scheme with the random resource allocation scheme (labeled as RRA) presented in [3] and resource allocation scheme with RF of 1 (labeled as RA with RF $=1$ ) presented in [11]. In RRA scheme, multiple D2D pairs residing in a cell can reuse the uplink cellular resources at the same time. However, due to coexistence of both cellular users and D2D pairs in same region of a cell imposed the aggregate interference. Whereas in the case of the RA with RF $=1$ scheme, allowing only one D2D pair to access a cellular resource at a time cannot accommodate high capacity of a system.

\subsubsection{Discussion on Performance of D2D Communications}

Here, we analyze the performance of D2D communications underlaying uplink cellular networks.

Figure 2 depicts the outage probability distribution of D2D communications at varying distance from the eNB to D2D-Rx. As expected, the D2D outage probability of the proposed scheme is much lower compared to the RRA scheme because a definite interference level is maintained. As can be seen, the outage probability increases due to the increase of uplink interference. In the RA with $\mathrm{RF}=1$ scheme, increasing the distance between D2D-Rx and eNB increases the outage probability significantly. It is observed that the analytical calculation of outage probability results of D2D is very close to the Monte Carlo simulation.

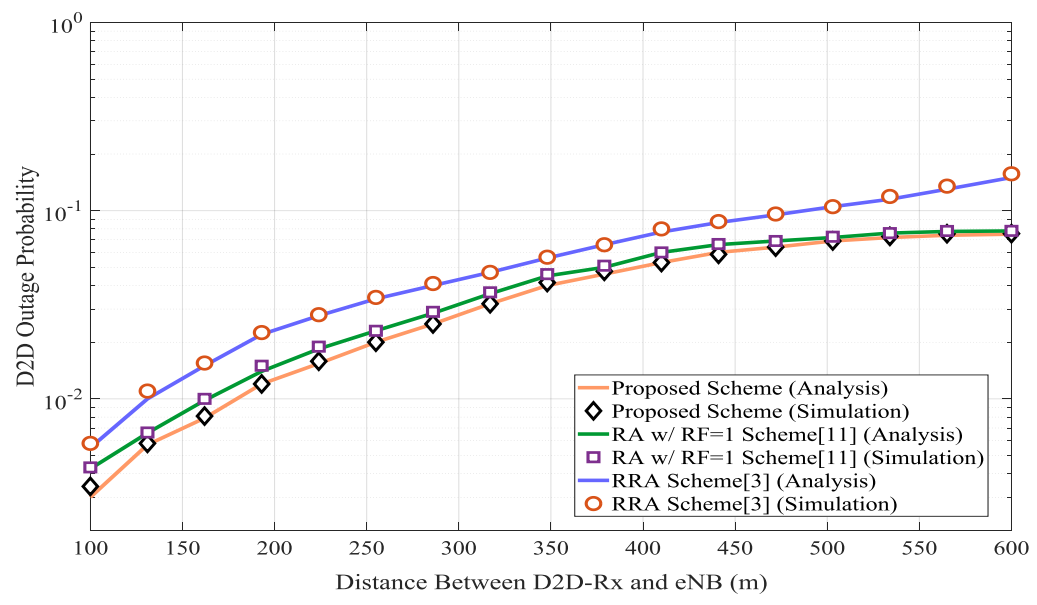

Figure 2. D2D outage probability with varying distance from eNB to D2D-Rx. 
Figure 3 depicts the outage probability distribution at varying SINR threshold value. We can see from Figure that the D2D outage probability increases with the increase of threshold value of SINR. A significant lower outage probability is observed at the SINR threshold value of $-10 \mathrm{~dB}$. It is observed that the proposed scheme experiences much lower outage probability than existing schemes. Comparing to the existing schemes, resource allocation and power control scheme with FRF of 2 yields a $20 \%$ reduction in outage probability. This is because of the fact that D2D-Tx in the proposed scheme maintains upper and lower bounds transmit power. We notice that the analytical calculation result is very close to that from the simulation result.

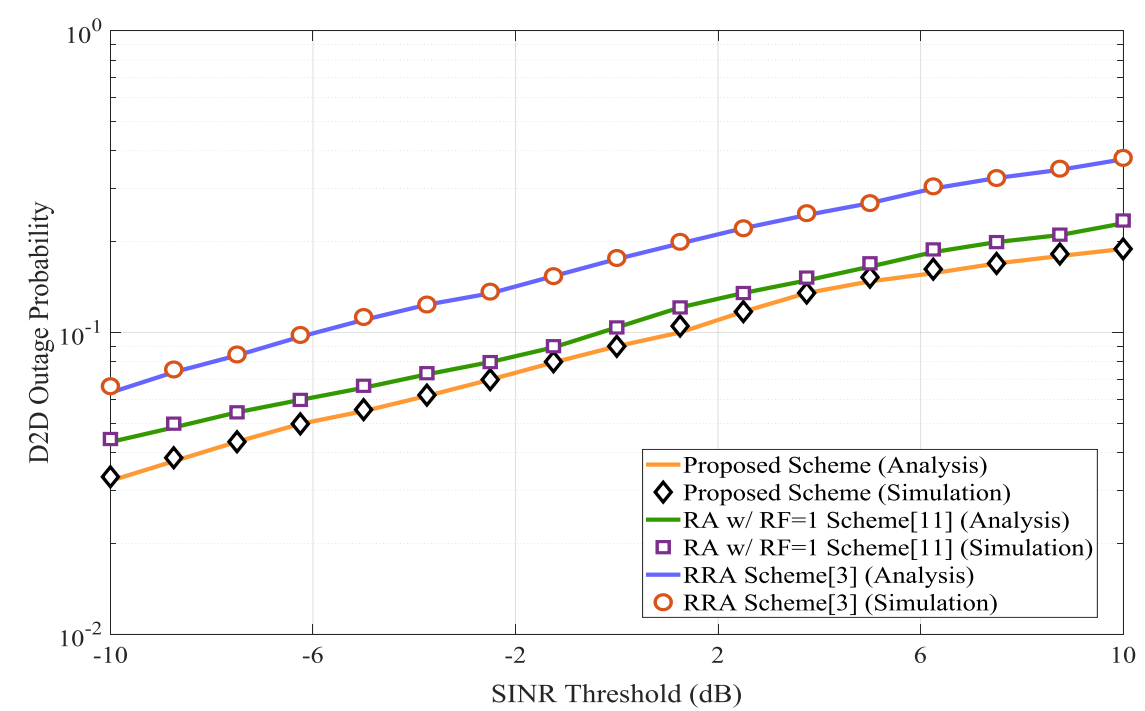

Figure 3. D2D outage probability with varying signal-to-interference-plus-noise ratio (SINR) threshold.

Figure 4 presents the D2D throughput at varying number of available D2D pairs in a cell. We can see from the Figure that the throughput increases with the increase of D2D pairs. Our proposed scheme yields highest throughput as compared with other existing schemes. This is because of the fact that the proposed scheme dramatically reduced the outage probability. By using our proposed scheme, data traffic through the cellular users is control; hence it maximizes the success rate of transmission. Enabling D2D communications with cellular resource RF of 2 achieves a 4.43 gain in the D2D throughput as compared with RRA scheme. Moreover, our proposed scheme yields a 1.5 gain in the D2D throughput as compared with RA with FRF of 1.

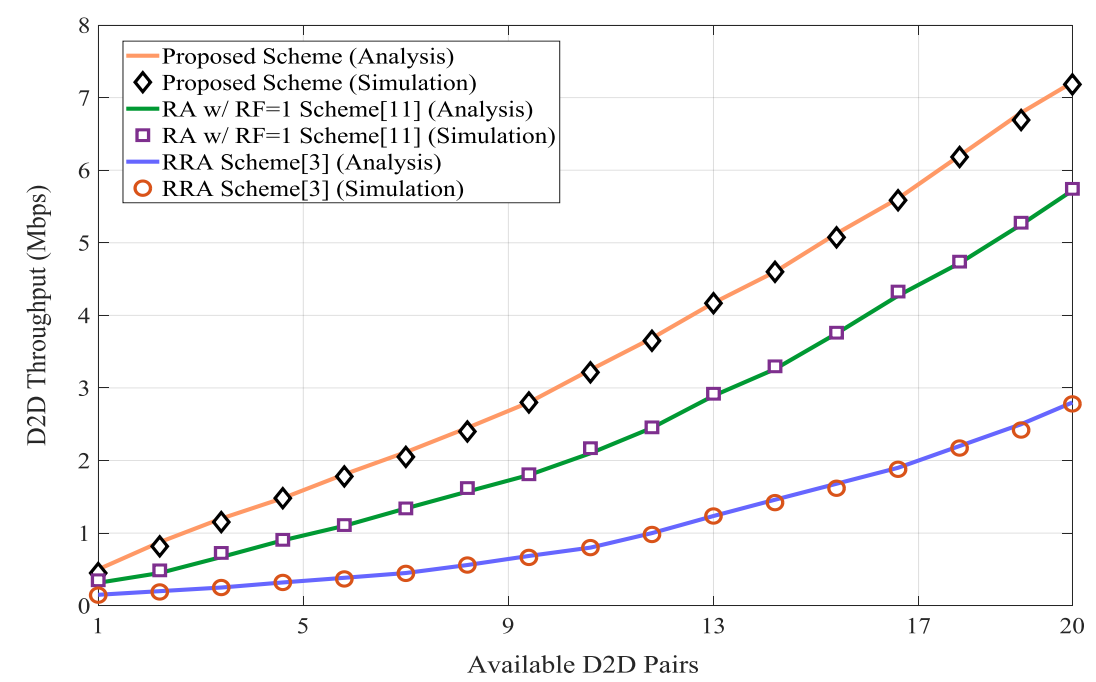

Figure 4. D2D throughput with varying number of available D2D pairs. 


\subsubsection{Discussion on Overall System Performance}

Figure 5 depicts the outage probability of the overall system for different values of distance from the eNB to D2D-Rx. When the distance increases, the channel gain decreases and thus increases outage probability. The significance of the proposed scheme is that, because of the FFR scheme with FRF of 2, the proposed scheme provides high utilization of resources within the distance of $100 \mathrm{~m}$. Thus, the proposed scheme helps to reduce the outage probability by minimizing uplink interference. However, RRA scheme dramatically increases the outage probability with the increase of distance due to the expansion of path-loss. This results in degradation of SINR.

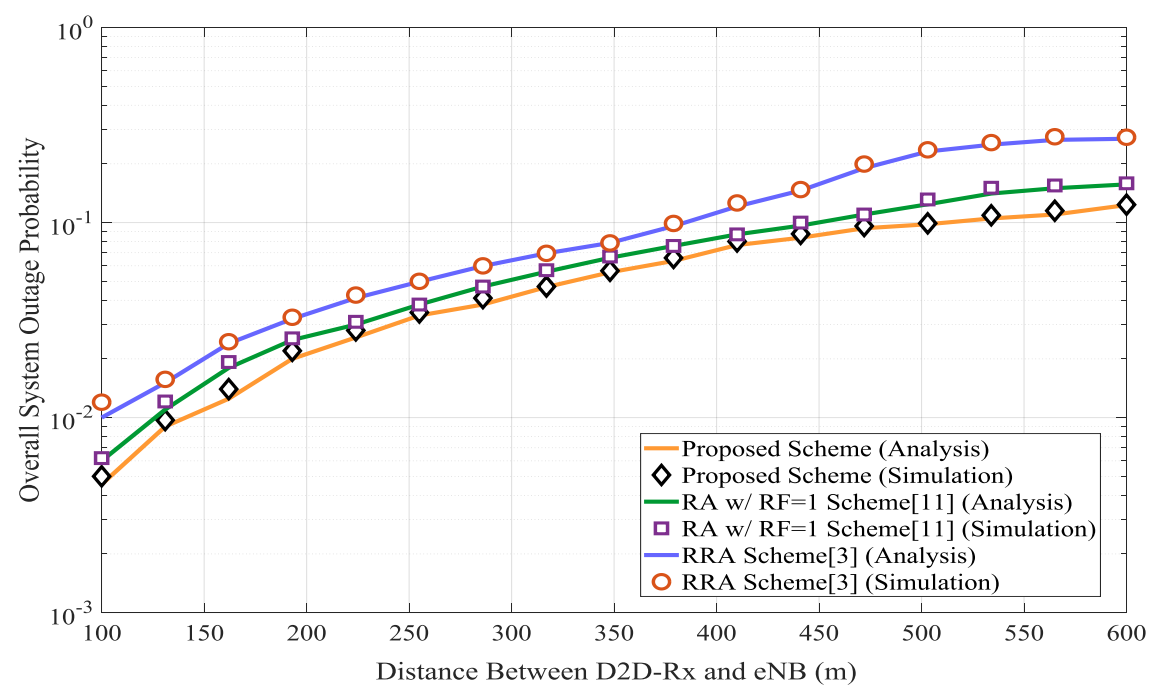

Figure 5. Overall system outage probability with varying distance from eNB to D2D-Rx.

In Figure 6, the outage probability of the system under varying threshold SINR is shown. The value of definite value of SINR threshold is varied from $-15 \mathrm{~dB}$ to $25 \mathrm{~dB}$. The proposed scheme has very low outage probability as compared to other existing schemes. It can be seen that, with the SINR threshold of $17 \mathrm{~dB}$, the system outage value for the proposed scheme, FRF of 1 scheme, and RRA scheme are $0.0989,0.125$, and 0.20 , respectively. This implies that the proposed scheme minimizes outage probability by means of joint resource allocation and power control method.

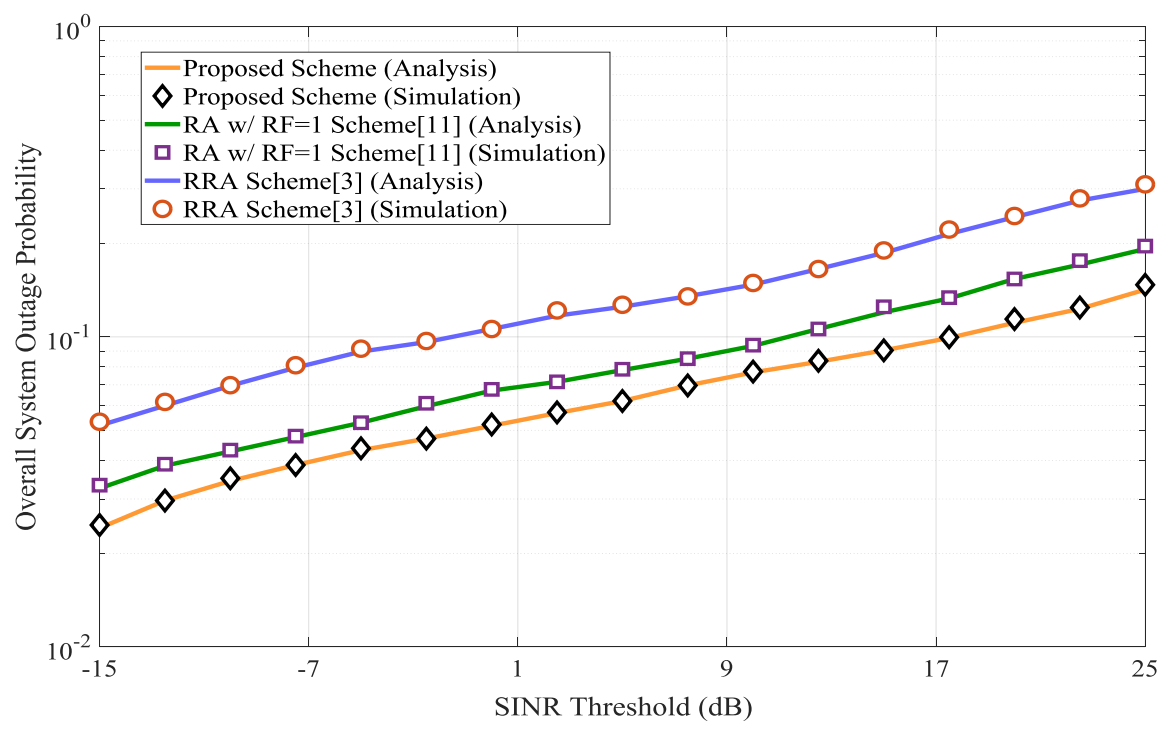

Figure 6. System outage probability with varying SINR threshold. 
Performance result for the system throughput at varying number of available D2D pairs is depicted in Figure 7. We observed that with the increase of D2D pairs, RA scheme achieved very less throughput because there is high rate of interference; in contrast, the proposed scheme yields highest throughput by maximizing the system capacity. Comparing to RA scheme, our proposed scheme provides a 4.38 throughput gain as compared with RRA scheme and a 1.45 throughput gain as compared with RA with FRF of 1 . The throughput gain of D2D communications at maximum number of available D2D pairs is higher than that of the overall system throughput; this is because as the D2D pairs increases cellular user throughput decreases.

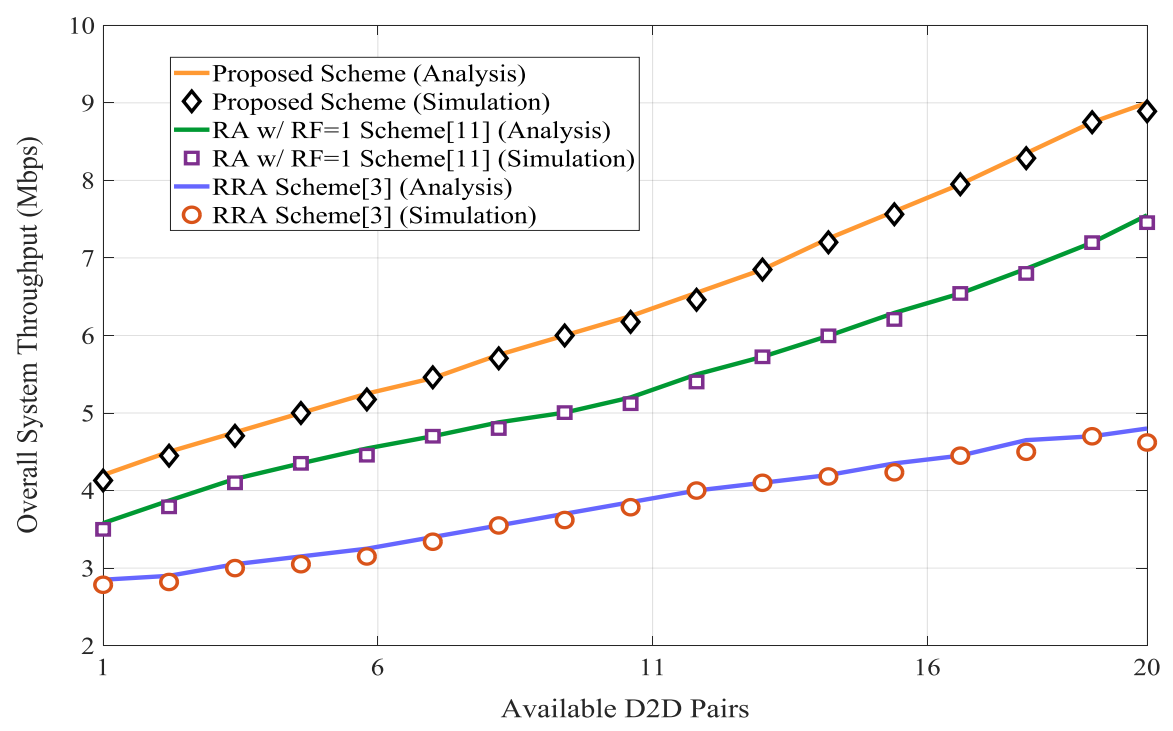

Figure 7. System outage probability with varying number of available D2D pairs.

In Figure 8, the throughput of the D2D users and cellular users over varying cellular user coverage distance. As expected, throughput of the D2D users is decreases with the increase of cellular coverage distance. This is because of the fact that D2D-Rx received more uplink interference from cellular users. However, as the cellular coverage distance increases throughout of the cellular users increases. It is observed that cellular user in the proposed scheme can afford more capacity as compared with other existing schemes.

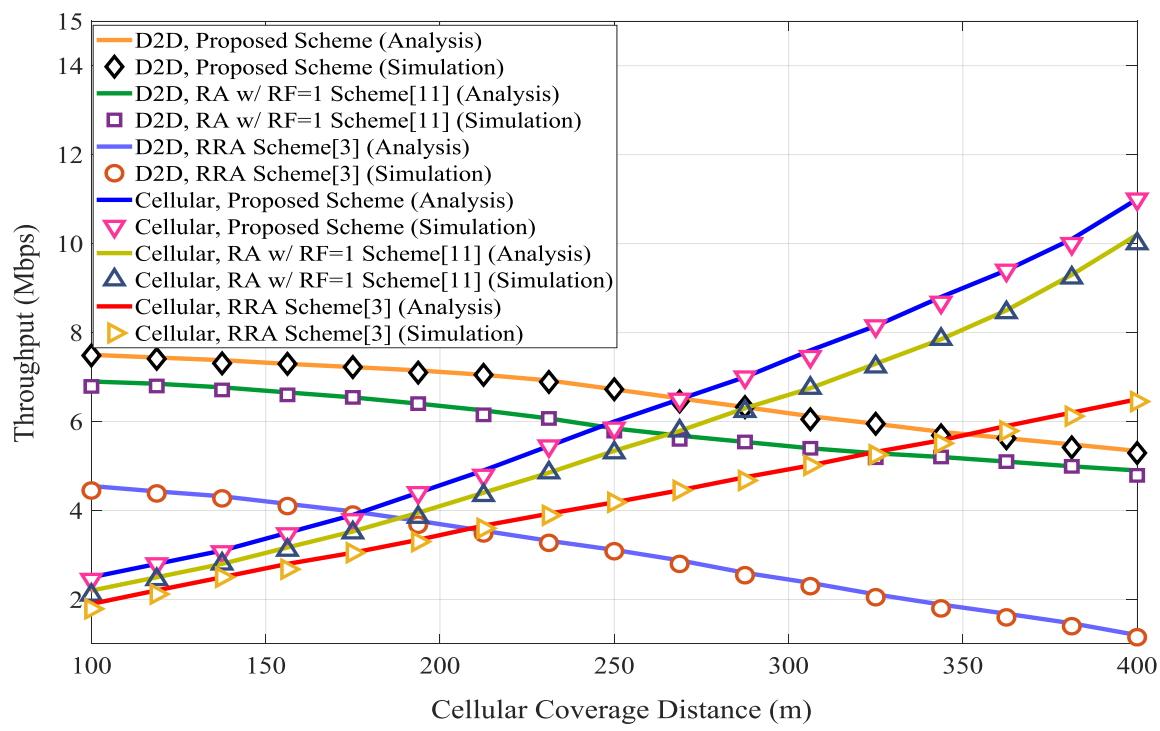

Figure 8. Throughput of D2D user and cellular user at varying cellular user coverage distance. 
Figure 9 shows the outage probability of cellular user over varying number of available D2D pairs at different SINR threshold values. It is seen from the Figure that the outage probability at threshold SINR value of $10 \mathrm{~dB}$ is higher than other values. Due to the increase of threshold SINR value, system capacity decreases dramatically.

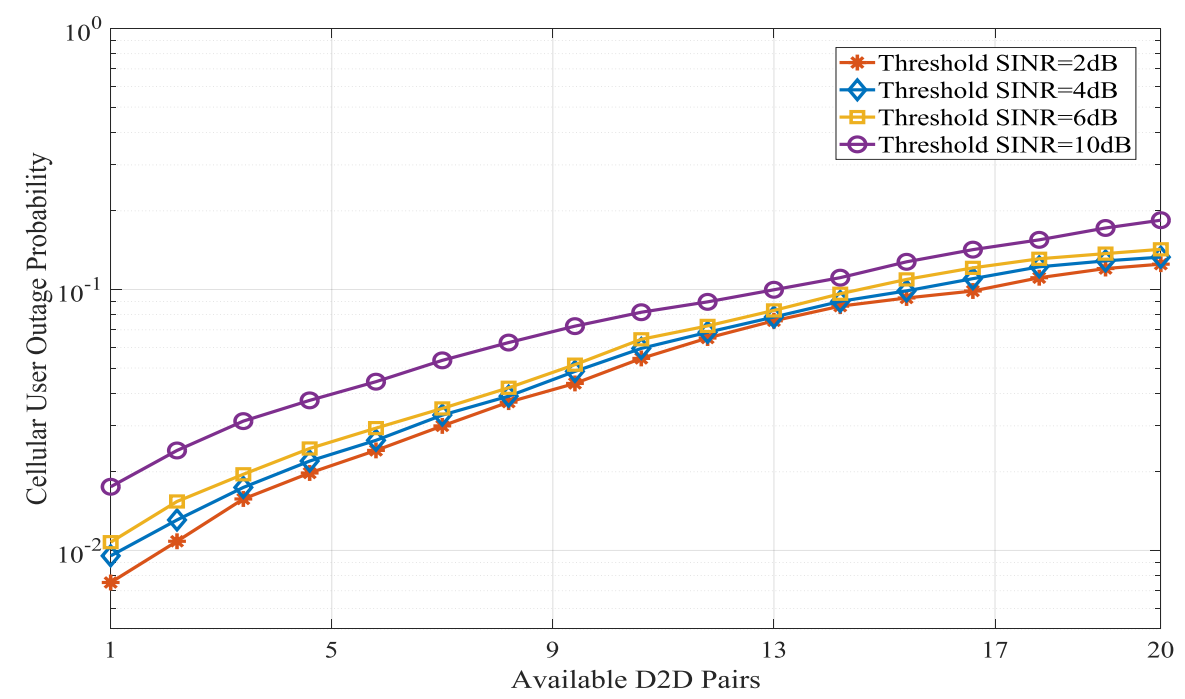

Figure 9. Cellular outage probability over available D2D pairs with varying threshold SINR.

The average energy consumption of the overall system with varying number of D2D pairs is shown in Figure 10. Our result shows that with the increase of number of D2D users, power consumption is increases. The worse case is the RA scheme which has upper bound of energy consumption. This is due to the fact that RA scheme could not reduce the uplink interference and this leads to subcarrier synchronization error. However, the energy consumption of the proposed scheme is very less as compared with other existing schemes. During the discovery of D2D pairs which can simultaneously share the same cellular resource, optimal synchronization of subcarriers is achieved by the proposed scheme. Therefore, our proposed scheme increases the success rate of transmission and is favorable for low-energy devices.

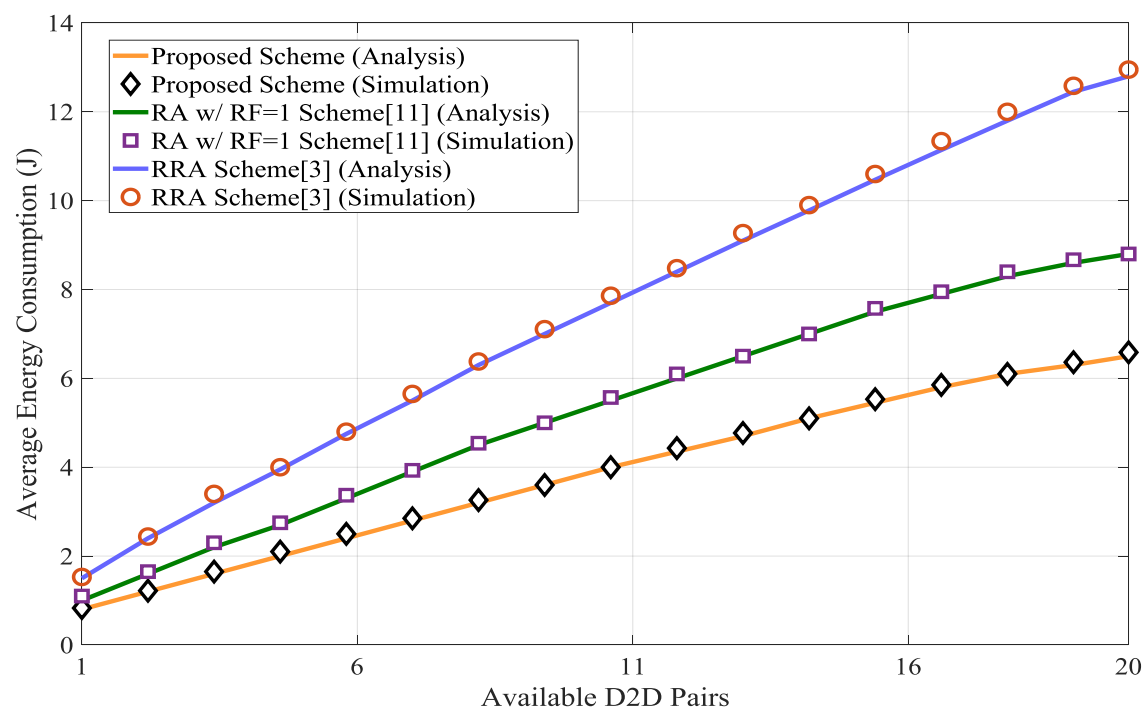

Figure 10. Average energy consumption of system with varying number of available D2D pairs. 


\section{Conclusions}

To deal with the ever increasing traffic through the cellular users, D2D communications has been considered as one of the promising technique. We studied the problem of non-orthogonal resource sharing between cellular users and D2D pairs in an uplink network to enable traffic offloading from eNB. In the paper, two-pairs of D2D users simultaneously shared the same uplink cellular resource. This introduced severe interference between the cellular users and D2D users. In order to minimize the resource reuse interference, a resource allocation and power control scheme using FFR method with FRF of 2 was proposed. Outage probability has become an imperative topic in cellular networks as it determines the capacity of the network. Based on the distances, the outage probability was calculated by ensuring SINR requirements of cellular users and D2D pairs. Moreover, the proposed scheme analyzed channel coefficient relationship based on the distances. We evaluated D2D throughput and overall system throughput mathematically and simulated using Monte Carlo simulation. Moreover, we analyzed the fair allocation of cellular resource among two D2D pairs by using Jain's fairness index. Precisely it is showed that the proposed scheme leverages higher system throughput by minimizing system outage value. Our analytical and simulation results showed a promising solution for accommodating demands for high data rate services. In future research, we will extend our work into the D2D communication scenario with large reuse factor.

Author Contributions: D.D.N. organized and developed the proposal of the study, carried out the mathematical analysis, and performed simulations using Monte Carlo. S.S. provided guidance, key suggestions, and finalized the paper.

Funding: This research is partly supported by Basic Science Research Program through the National Research Foundation of Korea (NRF) funded by the Ministry of Education with Grant No. NRF-2015R1D1A1A01059962 and a part of the project titled 'Development of Automatic Identification Monitoring System for Fishing Gears', funded by the Ministry of Oceans and Fisheries, Korea with Grant No. 20170388.

Conflicts of Interest: The authors declare no conflict of interest.

\section{References}

1. Li, Q.; Hu, R.Q.; Qian, Y.; Wu, G. Cooperative communications for wireless networks: techniques and applications in LTE-advanced systems. IEEE Wirel. Commun. 2012, 19, $22-29$.

2. Gandotra, P.; Jha, R.K. Device-to-Device communication in cellular networks: A Survey. J. Netw. Comput. Appl. 2016, 71, 99-117. [CrossRef]

3. Asadi, A.; Wang, Q.; Mancuso, V. A survey on Device-to-Device communication in cellular networks. IEEE Commun. Surv. Tutor. 2014, 16, 1801-1819. [CrossRef]

4. Noura, M.; Nordin, R. A survey on interference management for Device-to-Device (D2D) communication and its challenges in 5G networks. J. Netw. Comput. Appl. 2016, 71, 130-150. [CrossRef]

5. Safdar, G.A.; Ur-Rehman, M.; Muhammad, M.; Imran, M.A.; Tafazolli, R. Interference mitigation in D2D communication underlaying LTE-A network. IEEE Access 2016, 4, 7967-7987. [CrossRef]

6. Tsai, A.-H.; Wang, L.-C.; Huang, J.-H.; Lin, T.-M. Intelligent resource management for Device-to-Device (D2D) communications in neterogeneous networks. In Proceedings of the 15th International Symposium on Wireless Personal Multimedia Communications, Taipei, Taiwan, 24-27 September 2012; pp. 75-79.

7. Li, C.; Li, B.; Lan, B.; Zhang, Y.; Wang, T. Uplink power control for Device to Device communication underlaying cellular networks. In Proceedings of the 8th International Conference on Communications and Networking in China (CHINACOM), Guilin, China, 14-16 August 2013; pp. 256-259.

8. Katsinis, G.; Tsiropoulou, E.E.; Papavassiliou, S. Joint resource block and power allocation for interference management in Device to Device underlay cellular networks: A game theoretic approach. Mob. Netw. Appl. 2017, 22, 539-552. [CrossRef]

9. Chen, D.; Jiang, T.; Zhang, Z. Frequency partitioning methods to mitigate cross-tier interference in two-tier femtocell networks. IEEE Trans. Veh. Technol. 2015, 64, 1793-1805. [CrossRef] 
10. Ningombam, D.D.; Pyun, J.; Hwang, S.; Shin, S. Fractional frequency reuse scheme for interference mitigation in device-to-device communication underlying LTE-A networks. In Proceedings of the 2017 51st Asilomar Conference on Signals, Systems, and Computers, Pacific Grove, CA, USA, 29 October-1 November 2017; pp. 1402-1406.

11. Rodziewicz, M. Outage Probability of Device-to-Device Communications in Frequency Reuse-1 Networks. Mob. Netw. Appl. 2017, 22, 1058-1064. [CrossRef]

12. Gupta, S.; Kumar, S.; Zhang, R.; Kalyani, S.; Giridhar, K.; Hanzo, L. Resource allocation for D2D links in the FFR and SFR aided cellular downlink. IEEE Trans. Commun. 2016, 64, 4434-4448. [CrossRef]

13. Li, X.; Shankaran, R.; Orgun, M.A.; Fang, G.; Xu, Y. Resource allocation for underlay D2D communication with proportional fairness. IEEE Trans. Veh. Technol. 2018, 67, 6244-6258. [CrossRef]

14. Katsinis, G.; Tsiropoulou, E.E.; Papavassiliou, S. Multicell interference management in Device to Device underlay cellular networks. Futur. Internet 2017, 9, 44-64. [CrossRef]

15. Shalmashi, S.; Miao, G.; Han, Z.; Slimane, S.B. Interference constrained device-to-device communications. In Proceedings of the 2014 IEEE International Conference on Communications (ICC), Maoming, China, 14-16 August 2014; pp. 5245-5250.

16. Chhorn, S.; Yoon, S.; Seo, S.; Kim, S.; Cho, C. Spectrum reuse schemes with power control for Device-to-Device communication in LTE-advanced cellular network. KSII Trans. Inter. Inf. Syst. 2015, 9, 4819-4834.

17. Wang, J.; Zhu, D.; Zhang, H.; Zhao, C.; Li, J.C.F.; Lei, M. Resource optimization for cellular network assisted multichannel D2D communication. Signal Process. 2014, 100, 23-31. [CrossRef]

18. SHI, H.; Prasad, R.V.; Onur, E.; Niemegeers, I.G.M.M. Fairness in wireless networks: issues, measures and challenges. IEEE Commun. Surv. Tutor. 2014, 16, 5-24.

19. Sediq, A.B.; Gohary, R.H.; Yanikomeroglu, H. Optimal tradeoff between efficiency and Jain's fairness index in resource allocation. In Proceedings of the 2012 IEEE 23rd International Symposium on Personal, Indoor and Mobile Radio Communications-(PIMRC), Sydney, Australia, 9-12 September 2012; pp. 577-583.

20. Xiaoshuai, L.; Lin, M.; Yubin, X.; Shankaran, R. Joint power control and proportional fair scheduling for D2D communication underlaying cellular networks. In Proceedings of the 2016 IEEE 13th International Conference on Signal Processing (ICSP), Chengdu, China, 6-10 November 2016; pp. 1307-1312.

21. Huq, K.M.S.; Mumtaz, S.; Rodriguez, J. Outage probability analysis for device-to-device system. In Proceedings of the 2016 IEEE International Conference on Communications (ICC), Kuala Lumpur, Malaysia, 23-27 May 2016; pp. 1-5.

22. Ghavami, H.; Moghaddam, S.S. Analysis of outage probability for in-band Device-to-Device communications underlaying cellular network. Int. J. Commun. Syst. 2017, 30, e3286. [CrossRef]

23. IMT Vision-Framework and overall objectives of the future development of IMT for 2020 and beyond. Available online: https:/ / www.itu.int/rec/R-REC-M.2083 (accessed on 20 September 2018).

24. Wang, H.; Chu, X. Distance-constrained resource-sharing criteria for device-to-device communications underlaying cellular networks. Electron. Lett. 2012, 48, 528-530. [CrossRef]

25. Janis, P.; Koivunen, V.; Ribeiro, C.; Korhonen, J.; Doppler, K.; Hugl, K. Interference-aware resource allocation for Device-to-Device radio underlaying cellular networks. In Proceedings of the VTC Spring 2009-IEEE 69th Vehicular Technology Conference, Barcelona, Spain, 26-29 April 2009; pp. 1-5.

26. 3GPP TR36.814V0.4.1, Physical Layer Aspects for Evolved UTRA. Available online: http://www.3gpp.org. (accessed on 20 September 2018).

(C) 2018 by the authors. Licensee MDPI, Basel, Switzerland. This article is an open access article distributed under the terms and conditions of the Creative Commons Attribution (CC BY) license (http://creativecommons.org/licenses/by/4.0/). 